\title{
Bound or Free: Interaction of the C-Terminal Domain of Escherichia coli Single-Stranded DNA-Binding Protein (SSB) with the Tetrameric Core of SSB
}

Xun-Cheng Su, ${ }^{\dagger \neq}$ Yao Wang, ${ }^{\S}$ Hiromasa Yagi, ${ }^{\ddagger}$ Dmitry Shishmarev, ${ }^{\ddagger}$ Claire E. Mason, ${ }^{\S}$ Paul J. Smith, ${ }^{\ddagger}$ Marylène Vandevenne," Nicholas E. Dixon, ${ }^{\ddagger}$, and Gottfried Otting*,

\author{
${ }^{\dagger}$ State Key Laboratory of Elemento-Organic Chemistry, Nankai University, Tianjin 300071, China \\ ${ }^{\ddagger}$ Research School of Chemistry, Australian National University, Canberra, ACT 0200, Australia \\ ${ }^{\S}$ School of Chemistry and Centre for Medical and Molecular Bioscience, University of Wollongong, Wollongong, NSW 2522, \\ Australia \\ "School of Molecular Bioscience, University of Sydney, Sydney, NSW 2006, Australia
}

Supporting Information

\begin{abstract}
Single-stranded DNA (ssDNA)-binding protein (SSB) protects ssDNA from degradation and recruits other proteins for DNA replication and repair. Escherichia coli SSB is the prototypical eubacterial SSB in a family of tetrameric SSBs. It consists of a structurally well-defined ssDNA binding domain (OB-domain) and a disordered C-terminal domain (C-domain). The eight-residue C-terminal segment of SSB (C-peptide) mediates the binding of SSB to many different SSB-binding proteins. Previously published nuclear magnetic resonance (NMR) data of the monomeric state at $\mathrm{pH} 3.4$ showed that the C-peptide binds to the OB-domain at a site that overlaps with the ssDNA binding site, but investigating the protein at neutral $\mathrm{pH}$ is difficult because of the high molecular mass and limited

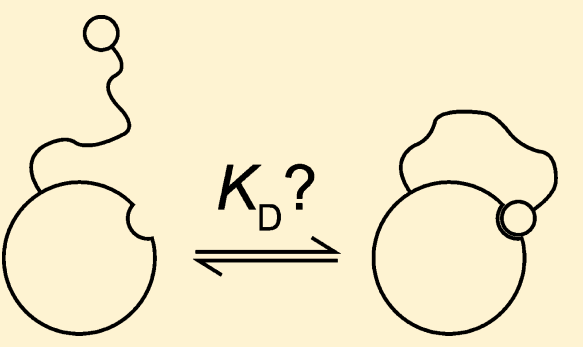
solubility of the tetramer. Here we show that the C-domain is highly mobile in the SSB tetramer at neutral pH and that binding of the C-peptide to the OB-domain is so weak that most of the C-peptides are unbound even in the absence of ssDNA. We address the problem of determining intramolecular binding affinities in the situation of fast exchange between two states, one of which cannot be observed by NMR and cannot be fully populated. The results were confirmed by electron paramagnetic resonance spectroscopy and microscale thermophoresis. The C-peptide-OB-domain interaction is shown to be driven primarily by electrostatic interactions, so that binding of 1 equiv of $(\mathrm{dT})_{35}$ releases practically all C-peptides from the OB-domain tetramer. The interaction is much more sensitive to $\mathrm{NaCl}$ than to potassium glutamate, which is the usual osmolyte in $E$. coli. As the Cpeptide is predominantly in the unbound state irrespective of the presence of ssDNA, long-range electrostatic effects from the Cpeptide may contribute more to regulating the activity of SSB than any engagement of the C-peptide by the OB-domain.
\end{abstract}

ingle-stranded DNA-binding proteins (SSBs) are essential $\checkmark$ proteins found in all kingdoms of life. SSB protects ssDNA from degradation and recruits other proteins required for DNA replication, recombination, and repair to ssDNA. ${ }^{1,2}$ Escherichia coli SSB is the prototype of prokaryotic tetrameric SSBs. ${ }^{3}$ It contains two domains, a structurally well-defined ssDNAbinding domain [oligonucleotide-binding domain (OB-domain)] and a C-terminal domain of unknown structure [Cdomain (Figure 1)]. ${ }^{4}$ The OB-domain is involved in ssDNA binding through electrostatic and hydrophobic interactions. ${ }^{5-7}$ Through association of four OB-domains, SSB forms a stable homotetramer that is stabilized by the presence of intact Cdomains. $^{8,9}$

ssDNA binds to the SSB tetramer in a strongly salt dependent manner. At low salt concentrations, binding of ssDNA shows a strong negative cooperativity so that only one oligo-deoxythymidine 35 mer $\left[(\mathrm{dT})_{35}\right]$ molecule binds to a SSB tetramer at $1.5 \mathrm{mM} \mathrm{NaCl}\left(25^{\circ} \mathrm{C}\right.$ and $\left.\mathrm{pH} 8.1\right)$ with high affinity, while a second $(\mathrm{dT})_{35}$ molecule does not bind even at
10 -fold higher DNA concentrations. ${ }^{10}$ This binding mode, in which only two of the OB-domains in the tetramer are loaded with ssDNA, is commonly termed the (SSB) 35 mode. ${ }^{4}$ Native ESI-MS experiments using different ratios of $(\mathrm{dT})_{35}$ to SSB tetramer confirmed that the 2:1 complex forms only if $(\mathrm{dT})_{35}$ is present at a molar ratio of $>1: 1{ }^{9}$ The 2:1 complex can serve as a model for the (SSB $)_{65}$ mode, in which ssDNA occupies all OB-domains.

Several X-ray crystal structures of tetrameric SSB from different organisms have been determined with and without ssDNA. $^{11-19}$ For constructs including the C-domain, its electron density has been invisible or observable only for sections that displayed considerable conformational variability, irrespective of the presence ${ }^{13,15,17}$ or absence of ssDNA. ${ }^{12,14} \mathrm{~A}$

Received: February 12, 2014

Published: March 7, 2014 
A

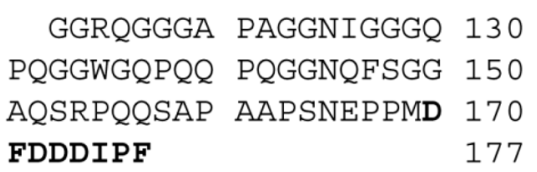

B

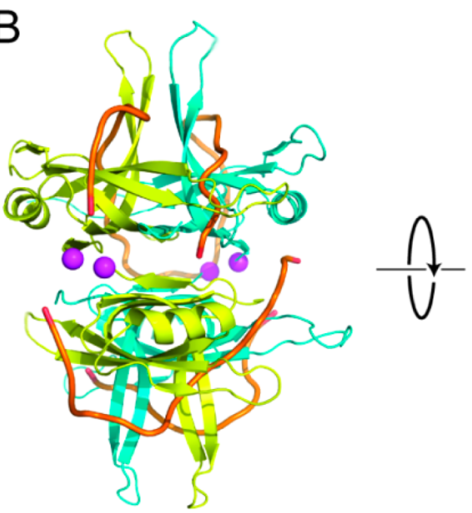

C

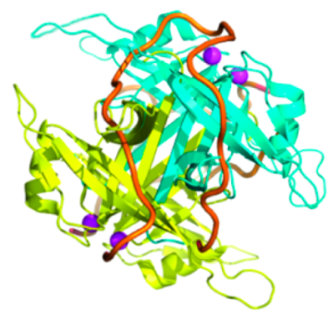

Figure 1. Amino acid sequence of the C-domain of E. coli SSB and structure of the OB-domain tetramer. (A) In this work, the C-domain is defined as residues $113-177$ and the C-peptide as residues 170-177 (bold). (B) Crystal structure of the OB-domain tetramer (Protein Data Bank entry 1EYG). ${ }^{13}$ Two of the OB-domains are colored yellow and the other two turquoise. The two $(\mathrm{dC})_{35}$ molecules in the structure are traced by orange lines drawn through the coordinates of the phosphorus atoms. Magenta spheres mark the C-terminus of each of the four OB-domains (carbonyl carbon of Leu112). (C) Same as panel $\mathrm{B}$, following rotation by $90^{\circ}$ around the horizontal axis. The length of each $35 \mathrm{mer}$ is $\sim 75 \AA$, i.e., shorter than the average end-toend distance of a random-coil peptide chain with 64 residues as in the C-domain (>90 ̊̊).

flexibly disordered structure of the E. coli C-domain is predicted from its high content of Gly, Pro, and Gln residues (Figure 1A).

The eight C-terminal residues of E. coli SSB form a highly negatively charged polypeptide that, in all cases tested to date, produces the binding interaction that recruits SSB-binding proteins. ${ }^{1,20}$ Most of these residues are strictly conserved in bacterial SSB sequences. ${ }^{21}$ Hereafter, we refer to the C-terminal eight-residue peptide segment as the "C-peptide".

Biochemical experiments with C-terminally truncated E. coli SSB produced indirect evidence that the C-peptide binds to the OB-domain in competition with ssDNA.,22,23 Competition between an SSB C-domain and ssDNA for binding to an OBdomain has also been inferred for the phage $\mathrm{T} 7$ gene 2.5 protein, a homodimeric SSB homologue. ${ }^{24}$ The competition model proposes that the C-terminal tail of SSB switches between a bound state in the absence of ssDNA and an unbound state in its presence. ${ }^{23}$ Binding to the OB-domain would protect both the $\mathrm{C}$-peptide and the OB-domain from nonfunctional electrostatic interactions, whereas the unbound state would make the OB-domain accessible to ssDNA and the C-peptide available for binding to SSB-binding proteins. The number of C-terminal tails displayed by SSB tetramers is functionally important for survival. ${ }^{25}$

At low $\mathrm{pH}$ (3.4), the SSB tetramer separates into its monomeric components while maintaining the three-dimensional (3D) fold of the OB-domain. Under those conditions, we could show by nuclear Overhauser effects (NOEs) that the Cpeptide binds to the OB-domain at a site that overlaps with the ssDNA binding site, while the rest of the C-domain is highly mobile. ${ }^{26}$ To judge the functional importance of this interaction, however, it is important to determine the affinity between the C-peptide and OB-domain for tetrameric SSB under physiological conditions. This measurement is not trivial, as intramolecular affinities cannot be determined by titration experiments; instead, the affinities require the determination of the populations of bound and free states in the unmodified system. In the case of SSB, NMR signals of the OB-domain tetramer are difficult to observe because of the high molecular mass ( $50 \mathrm{kDa}$ for the four OB-domains) and poor solubility of the tetramer.

Herein, we present an analysis of the affinity of the Cpeptide-OB-domain interaction to address two questions. (i) In the absence of ssDNA, is the C-peptide mostly bound to the OB-domain or free in solution? (ii) Does the binding of 1 equiv of $(\mathrm{dT})_{35}$ cause the release of all C-peptides from the OBdomain tetramer? The answers to these questions are fundamentally important for the hypothesis that binding of ssDNA to SSB increases the population of unbound C-peptide, thus acting as a signal for recruitment of SSB-binding proteins. To address these questions, we studied the C-domain-OBdomain interactions in the E. coli SSB tetramer at neutral $\mathrm{pH}$ by NMR spectroscopy, EPR spectroscopy, and microscale thermophoresis (MST) experiments in the absence and presence of ssDNA.

\section{EXPERIMENTAL PROCEDURES}

Sample Preparation and Purification. Construction of plasmids that direct overexpression of SSB $\Delta$ C62 (SSB-T*) and its K43C mutant and full-length SSB(K43C) and SSB(M169C) are described in Text $\mathrm{S} 1$ of the Supporting Information, along with a new method for purifying SSB $\Delta$ C62; SSB $\Delta$ C62(K43C) was prepared similarly. Uniformly isotope-labeled samples of $\mathrm{SSB}, \mathrm{SSB} \Delta \mathrm{C} 8$, and their cysteine mutants were produced as described previously, ${ }^{9,23}$ except that all buffers for the mutants were supplemented with $2 \mathrm{mM}$ dithiothreitol. The $\left[{ }^{13} \mathrm{C}\right]$ methionine-labeled samples of SSB were prepared in the same way, except that $200 \mathrm{mg} / \mathrm{L}\left[{ }^{13} \mathrm{C}\right]$ methionine was added to M9 medium containing glucose and ammonium chloride at natural isotopic abundance. SSB concentrations refer to the tetramer $\left(\mathrm{SSB}_{4}\right)$ throughout.

Labeling of SSB(M169C) with MTSL followed an established protocol ${ }^{27}$ in $50 \mathrm{mM}$ Tris- $\mathrm{HCl}(\mathrm{pH} 8.0)$ and 300 $\mathrm{mM} \mathrm{NaCl}$, in complete analogy with the labeling with $\left[{ }^{13} \mathrm{C}\right] \mathrm{MMTS}$ (Text S2 of the Supporting Information).

NMR Spectroscopy. All NMR measurements were conducted at $\mathrm{pH} 7.2$ and $25{ }^{\circ} \mathrm{C}$ unless stated otherwise. Data for resonance assignments of SSB were collected using $3 \mathrm{~mm}$ sample tubes in Bruker 600 and $800 \mathrm{MHz}$ NMR spectrometers equipped with TCI cryoprobes. All 3D NMR spectra were recorded in $20 \mathrm{mM}$ Tris- $\mathrm{HCl}(\mathrm{pH} \mathrm{7.2)}$ and $500 \mathrm{mM} \mathrm{NaCl}$. Backbone resonance assignments were obtained from the analysis of $3 \mathrm{D} \mathrm{CBCA}(\mathrm{CO}) \mathrm{NH}$ and HNCA spectra together with NOESY $-{ }^{15} \mathrm{~N}$ HSQC and two-dimensional (2D) TOCSY spectra of uniformly ${ }^{15} \mathrm{~N}$ - and ${ }^{13} \mathrm{C}$-labeled, ${ }^{15} \mathrm{~N}$-labeled, or unlabeled samples. The 3D NOESY $-{ }^{15} \mathrm{~N}$ HSQC spectrum used a mixing time of $100 \mathrm{~ms}$ and a $0.2 \mathrm{mM}$ solution of $\left[{ }^{15} \mathrm{~N}\right]$ SSB tetramer. 2D TOCSY spectra used a $0.25 \mathrm{mM}$ sample in $20 \mathrm{mM}$ Tris- $\mathrm{HCl}(\mathrm{pH} 7.2)$ and $300 \mathrm{mM} \mathrm{NaCl}$. Sample tubes of $5 \mathrm{~mm}$ diameter were used for measurements at low salt concentrations.

Relaxation Measurements. ${ }^{15} \mathrm{~N}$ relaxation data were recorded on the $600 \mathrm{MHz}$ NMR spectrometer, using standard 
pulse sequences ${ }^{28}$ and a $0.125 \mathrm{mM}$ solution of $\left[{ }^{15} \mathrm{~N}\right] \mathrm{SSB}$ tetramer in $20 \mathrm{mM}$ Tris- $\mathrm{HCl}$ at 300 and $500 \mathrm{mM} \mathrm{NaCl}$. The relaxation delays were $18,36,54,82,90,126$, and $142 \mathrm{~ms}$ in the $R_{2}$ experiment and 3,70,100,300,500,750, and $1000 \mathrm{~ms}$ in the $R_{1}$ experiment. The $\tau_{\mathrm{cp}}$ delay between the $\pi$ pulses of the CPMG sequence was $900 \mu \mathrm{s}$. The relaxation data were fit using Sparky. ${ }^{29}$

EPR Spectroscopy. X-Band EPR spectra of $10 \mu \mathrm{M}$ aqueous solutions of SSB(M169C) with a nitroxide (MTSL) tag on residue 169 were recorded. The spectra were recorded at room temperature using a Bruker 500E X-band EPR spectrometer with a microwave frequency of $9.807 \mathrm{GHz}$, a microwave power of $0.5 \mathrm{~mW}$, a modulation frequency of $100 \mathrm{kHz}$, a modulation amplitude of $0.5 \mathrm{G}$, and a time constant of $0.3 \mathrm{~s}$.

Microscale Thermophoresis. MST measurements of the binding affinity of SSB-Ct (amino acid sequence: PSNEPPMDFDDDIPF) for the OB-domain tetramer were performed at room temperature with fluorescein 5-maleimide (Thermo Scientific) conjugated with the cysteine residue in $\mathrm{K} 43 \mathrm{C}$ mutants of SSB. Measurements used three different preparations of the K43C mutant SSBs, including full-length SSB, SSB $\Delta \mathrm{C} 8$, and SSB $\Delta \mathrm{C} 62$. In each case, the fluorescently labeled mutant protein was exchanged ${ }^{9}$ with a 25 -fold excess of unconjugated SSB, SSB $\Delta$ C8, and SSB $\Delta$ C62, respectively. Thus, thermophoresis was detected essentially exclusively from mixed tetramers in which only one subunit is labeled with fluorescein, minimizing the influence of the fluorescent tag. The experiments used 50\% LED power (blue) and $90 \%$ MST power on a NanoTemper Monolith NT.115 instrument.

\section{RESULTS}

NMR Resonance Assignments. Backbone resonance assignments of the $\mathrm{C}$-domain were obtained under conditions of high salt ( 300 or $500 \mathrm{mM} \mathrm{NaCl}$ ) because SSB is only poorly soluble at low ionic strengths. ${ }^{8} 2 \mathrm{D}{ }^{1} \mathrm{H}-{ }^{1} \mathrm{H}$ TOCSY spectra showed many well-resolved spin systems, including the signals of six alanines (A120, A122, A151, A159, A161, and A162), two isoleucines (I126 and I175), and one tryptophan (W135) (Figure S1 of the Supporting Information). The observation of these residues in 2D TOCSY spectra despite the high molecular mass of the system $(75 \mathrm{kDa})$ attests to the high mobility of the C-domain. The small chemical shift dispersion of the amide protons confirms that the structure of the C-domain is predominantly disordered.

In agreement with the results from the TOCSY experiment, ${ }^{15} \mathrm{~N}$ HSQC cross-peaks were at least partially resolved for more than 40 of the 55 non-proline residues in the C-domain; 37 of these were sequence-specifically assigned to the $\mathrm{C}$-domain by triple-resonance 3D NMR experiments using uniformly ${ }^{15} \mathrm{~N}$ and ${ }^{13} \mathrm{C}$-labeled SSB (Figure 2). There was no evidence of cross-peaks for residues of the OB-domain of SSB in the ${ }^{15} \mathrm{~N}$ HSQC spectrum, consistent with the notion that the OBdomain forms a stable, structurally rigid tetramer with a molecular mass $(\sim 50 \mathrm{kDa})$ that is too high to yield observable ${ }^{15} \mathrm{~N}$ HSQC cross-peaks. The different motional regimes of the OB-domain tetramer and of the C-domains thus led to a greatly simplified NMR spectrum that selectively displays the resonances of the $\mathrm{C}$-domain.

All SSB samples at low ionic strengths proved to be sensitive to proteolytic digestion of the $\mathrm{C}$-domain, resulting in new intense cross-peaks after a few hours at $25^{\circ} \mathrm{C}$. In addition, SSB is not very soluble at low salt. We therefore restricted the

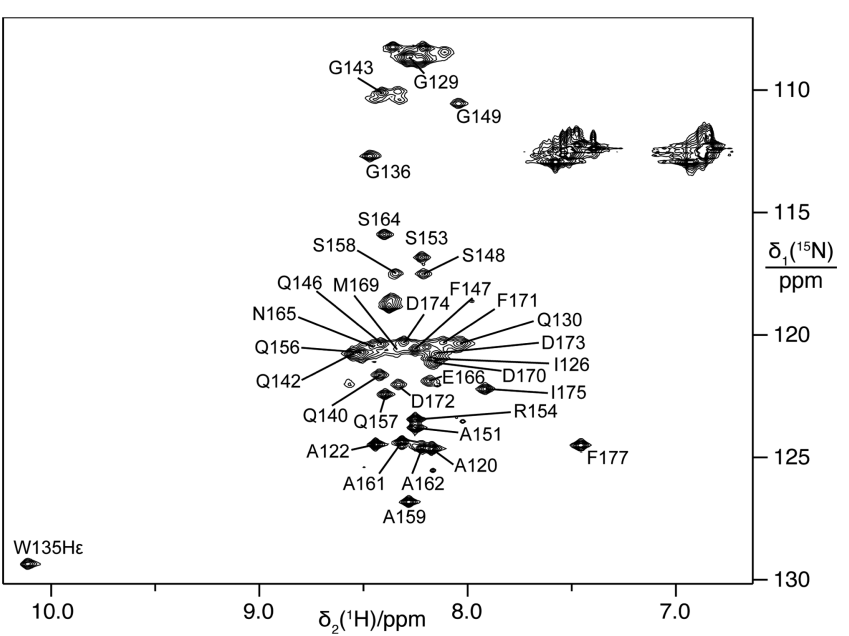

Figure 2. ${ }^{15} \mathrm{~N}$ HSQC spectrum of a $0.2 \mathrm{mM}$ solution of ${ }^{15} \mathrm{~N}$-labeled $E$. coli SSB tetramer in $20 \mathrm{mM}$ Tris- $\mathrm{HCl}(\mathrm{pH} 7.2)$ and $300 \mathrm{mM} \mathrm{NaCl}$ at $25{ }^{\circ} \mathrm{C}$. Concentrations of SSB are given in terms of tetramer $\left(\mathrm{SSB}_{4}\right)$ throughout. Assignments are shown for cross-peaks of backbone amides of the C-domain and the side chain $\mathrm{NH}$ of Trp135.

analysis at low salt concentrations to sensitive heteronuclear correlation spectra that could be recorded quickly. Fortunately, the ${ }^{15} \mathrm{~N}$ HSQC spectra at 10,300 , and $500 \mathrm{mM} \mathrm{NaCl}$ were very similar, with no chemical shift changes of $>0.12 \mathrm{ppm}$ between 10 and $300 \mathrm{mM} \mathrm{NaCl}$ (Figures S2A and S3A of the Supporting Information). This allowed the transfer of the NMR resonance assignments to the spectra obtained under low-salt conditions. Remarkably, ${ }^{15} \mathrm{~N}$ HSQC cross-peaks of the C-peptide were readily observed under low salt conditions, albeit with intensities that were weaker than those for the preceding residues. The $\mathrm{C}$-domain is thus highly mobile also at low salt concentrations, but the C-peptide is partly immobilized.

Effect of ssDNA. Titration of SSB with $(\mathrm{dT})_{35}$ at low salt concentrations resulted in the disappearance of the ${ }^{15} \mathrm{~N}$ HSQC cross-peaks of the C-peptide and the appearance of new crosspeaks at different chemical shifts (Figure 3 ). This shows that the exchange of $(\mathrm{dT})_{35}$ between different SSB tetramers is slow $\left(<100 \mathrm{~s}^{-1}\right)$, in agreement with earlier mass spectrometry results. ${ }^{9}$

The cross-peaks observed after the addition of a 2-fold excess of $(\mathrm{dT})_{35}$ to a fresh sample of SSB coincided with the crosspeaks observed after proteolysis of SSB (Figure S4 of the Supporting Information). Therefore, these conditions reflect the unbound state of the C-peptide.

Compared to SSB without ssDNA, the presence of $(\mathrm{dT})_{35}$ increased the signal intensities of the C-peptide, indicating its release from the OB-domain tetramer. The C-peptide also displayed the largest chemical shift changes (Figure S3B of the Supporting Information), confirming the previous results obtained at $\mathrm{pH} 3.4$ that showed that the C-peptide drives the interaction between the $\mathrm{C}$-domain and the OB-domain. ${ }^{26}$ The heterogeneous appearance of the NMR line shapes of the 1:1 complex $\left[(\mathrm{dT})_{35}: \mathrm{SSB}_{4}\right]$ is expected, as a single $(\mathrm{dT})_{35}$ molecule can occupy only half of the ssDNA binding sites of the SSB tetramer, generating different chemical environments for the $\mathrm{C}$ domains from different SSB protomers.

The cross-peaks of the C-domain in the 2:1 complex (not shown) overlapped with those of the 1:1 complex, indicating that the C-peptide is almost completely unbound already in the 1:1 complex. Addition of $(\mathrm{dT})_{35}$ beyond a 2:1 ratio did not 
A

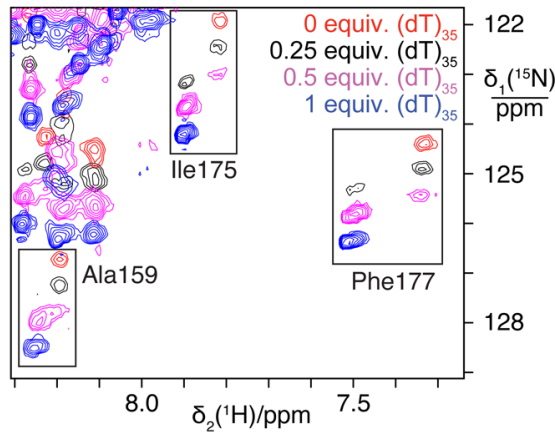

B

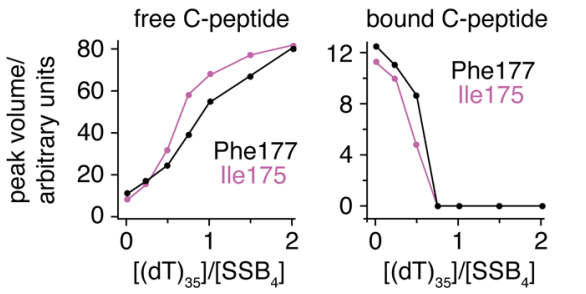

Figure 3. Titration of SSB with $(\mathrm{dT})_{35}$. (A) Overlay of ${ }^{15} \mathrm{~N}$ HSQC spectra of a $8.5 \mu \mathrm{M}$ solution of the ${ }^{15} \mathrm{~N}$-labeled SSB tetramer at low salt concentrations $[10 \mathrm{mM} \mathrm{NaCl}$ and $20 \mathrm{mM}$ Tris- $\mathrm{HCl}(\mathrm{pH} 7.2)]$ recorded with increasing amounts of $(\mathrm{dT})_{35}$. For improved visualization, the spectrum without $(\mathrm{dT})_{35}$ was plotted at the correct position, while the spectra with $(\mathrm{dT})_{35}$ were overlaid with a systematic displacement in the ${ }^{15} \mathrm{~N}$ dimension of $\sim 0.7 \mathrm{ppm}$ from spectrum to spectrum. Frames identify the cross-peaks of Ala159, Ile175, and Phe177 in the different spectra. All spectra were processed identically and plotted with the same contour levels. With increasing amounts of ssDNA, the intensities of the original cross-peaks decrease, while new cross-peaks appear for the 1:1 complex. The cross-peaks of the 1:1 complex display inhomogeneous line shapes. (B) Cross-peak volumes of the backbone amides of Ile175 and Phe177 during titration with $(\mathrm{dT})_{35}$. The left and right panels show the cross-peak intensities of the complex with ssDNA and of free SSB, respectively. The cross-peak intensities remained unaltered at titration ratios of 3:1 and 4:1 $\left[(\mathrm{dT})_{35}: \mathrm{SSB}_{4}\right]$. The data in panel $\mathrm{B}$ were recorded specifically to demonstrate the sigmoidal shapes of the titration curves that are evidence of cooperative release of the C-peptide from the SSB tetramer. In contrast to the spectra in panel $\mathrm{A}$, the sample used for the spectra in panel B showed weak cross-peaks of the ssDNA-SSB complex already prior to the addition of $(\mathrm{dT})_{35}$, which was attributed to small amounts of chromosomal ssDNA fragments copurified with SSB.

result in further changes in the signal intensities or chemical shifts of SSB.

Effect of $\mathrm{NaCl}$ and Potassium Glutamate. Compared with the low-salt spectra, the NMR signals in the presence of $300 \mathrm{mM} \mathrm{NaCl}$ were systematically shifted toward the chemical shifts of the unbound C-peptide (Figure S2A of the Supporting Information), and the largest chemical shift changes were observed for the resonances of the C-peptide (Figure S3A of the Supporting Information). The intensities of its signals also increased significantly (Figure S2A of the Supporting Information), as expected for enhanced mobility. Therefore, salt liberates the bound C-peptide from its binding site on the OB-domain tetramer. Our previous work with monomeric SSB at $\mathrm{pH} 3.4$ showed that the negatively charged $\mathrm{C}$-peptide binds to a positively charged binding site on the OB-domain that overlaps with the binding site of ssDNA. ${ }^{26}$ Salt is expected to weaken this electrostatic interaction. As salt induced smaller shifts than $(\mathrm{dT})_{35}$, it is less effective at breaking the C-peptideOB-domain interaction.
Glutamate is much more abundant in the E. coli cytosol than chloride. ${ }^{30}$ Therefore, we also recorded ${ }^{15} \mathrm{~N}$ HSQC spectra of SSB in the presence of potassium glutamate; 100 and $300 \mathrm{mM}$ potassium glutamate attenuated the peak intensities in the ${ }^{15} \mathrm{~N}$ HSQC spectrum without changing their chemical shifts (data not shown). As the C-peptide is in fast exchange between bound and free states (see below), the conservation of chemical shifts shows that glutamate hardly affects the C-peptide-OBdomain interaction in free SSB.

${ }^{15} \mathrm{~N}$ Relaxation. To increase sensitivity, ${ }^{15} \mathrm{~N}$ relaxation measurements were performed with more concentrated samples in the presence of 300 and $500 \mathrm{mM} \mathrm{NaCl}$. The Cpeptide displayed clearly enhanced transverse relaxation rates $\left(R_{2}\right)$ at $300 \mathrm{mM}$ but not at $500 \mathrm{mM} \mathrm{NaCl}$ (Figure 4B),
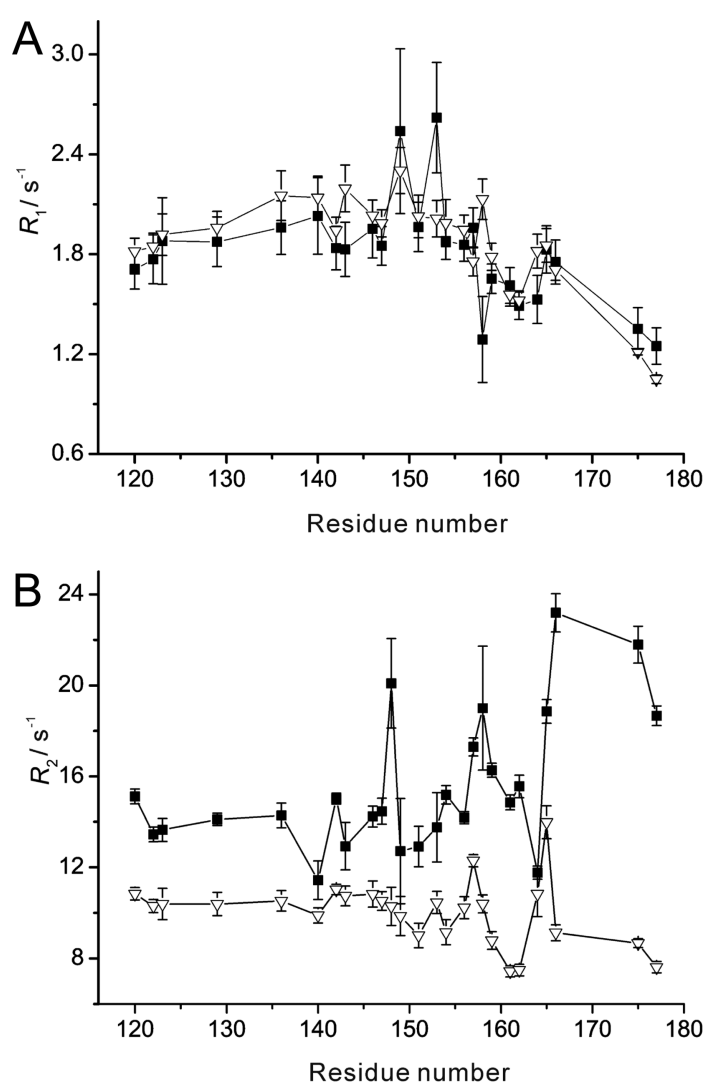

Figure $4 .{ }^{15} \mathrm{~N}$ relaxation shows the release of the C-peptide at high salt concentrations. (A) Longitudinal and (B) transverse ${ }^{15} \mathrm{~N}$ relaxation rates of $0.125 \mathrm{mM}\left[{ }^{15} \mathrm{~N}\right] \mathrm{SSB}_{4}$ in $20 \mathrm{mM}$ Tris- $\mathrm{HCl}(\mathrm{pH} 7.2)$ with 300 $(\boldsymbol{\square})$ and $500 \mathrm{mM} \mathrm{NaCl}(\nabla)$.

indicating that $300 \mathrm{mM} \mathrm{NaCl}$ is not sufficient to abolish all interactions between the C-peptide and the core of the tetramer. The overall decrease in $R_{2}$ rates observed throughout the C-domain at $500 \mathrm{mM} \mathrm{NaCl}$ suggests that all of the Cdomain becomes more mobile when the C-peptides no longer interact with the OB-domains. In contrast to $R_{2}$, the longitudinal relaxation rates $\left(R_{1}\right)$ are not sensitive to small populations of the immobilized C-peptide (Figure 4A). The $R_{1}$ rates decrease toward the C-terminus of SSB, as expected for an overall increase in mobility in an already flexible polypeptide chain.

Fast Exchange between the Bound and Free CPeptide. To determine the fraction of the bound C-peptide under low-salt conditions in the absence of ssDNA, it is 

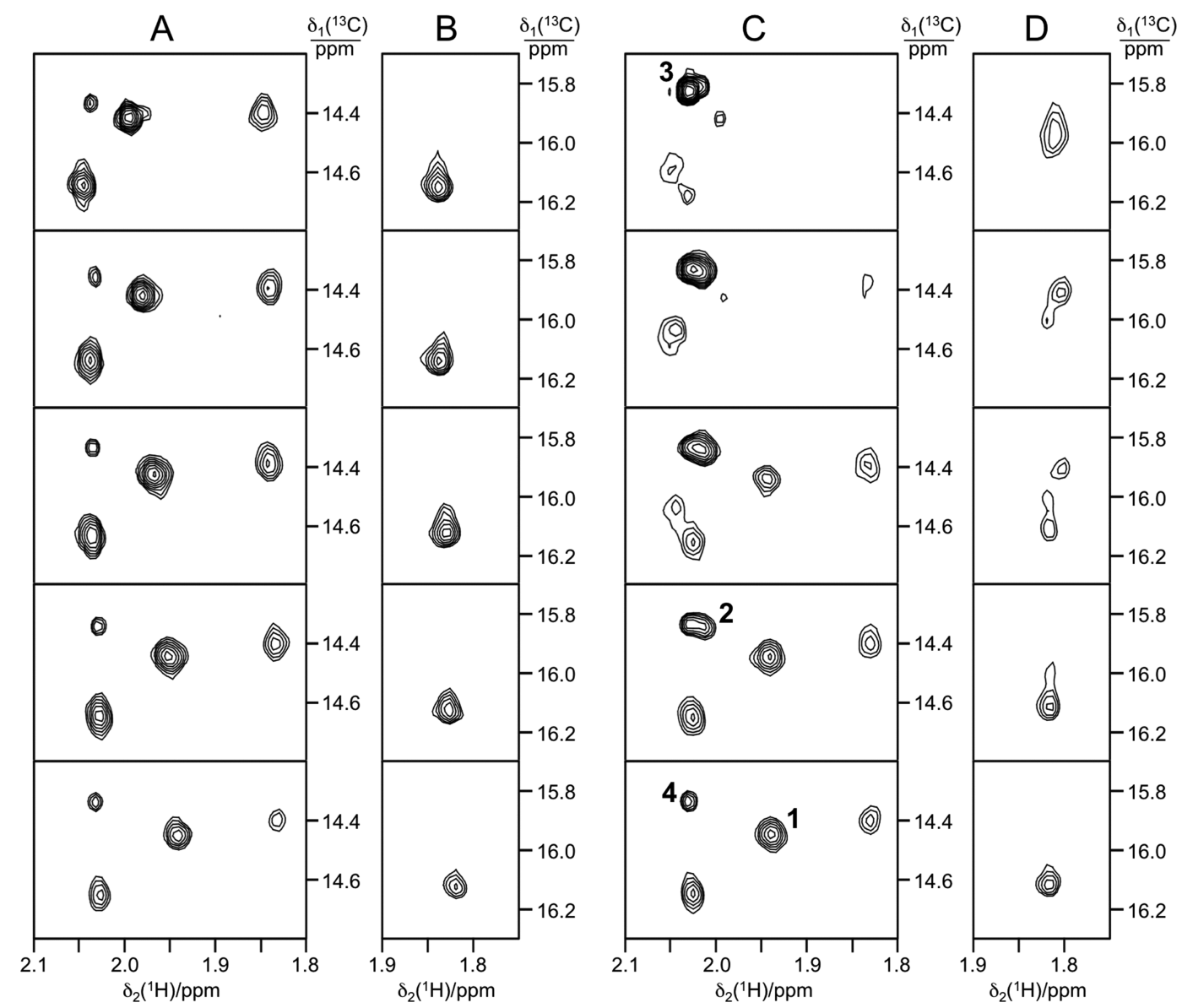

Figure 5. $\mathrm{NaCl}$ and $(\mathrm{dT})_{35}$ release the $\mathrm{C}$-peptide from the OB-domain tetramer. The figure shows ${ }^{13} \mathrm{C}$ HMQC spectra of $8.5 \mu \mathrm{M}$ solutions of $\left[{ }^{13} \mathrm{C}\right]$ methionine-labeled $\mathrm{SSB}_{4}$ in $20 \mathrm{mM}$ Tris- $\mathrm{HCl}$ (pH 7.2). (A) Titration with $\mathrm{NaCl}(10,50,100,200$, and $300 \mathrm{mM}$ from bottom to top,

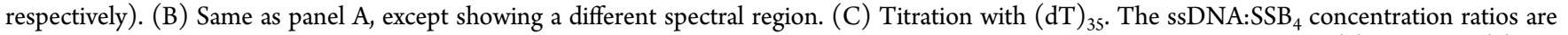
$0,0.25,0.5,1$, and 2 from bottom to top, respectively. The labeled cross-peaks represent Met169 in different states of SSB: (1) free SSB, (2) 1:1 complex of $(\mathrm{dT})_{35}$ with $\mathrm{SSB}_{4}$, (3) 2:1 complex of $\left(\mathrm{dT}_{35}\right.$ with $\mathrm{SSB}_{4}$, and (4) impurity attributed to SSB in complex with E. coli ssDNA fragments. (D) Same as panel C, except showing a different spectral region.

necessary to establish whether the equilibrium between the bound and unbound C-peptide is fast or slow on the NMR time scale. In the limit of fast exchange, the weak intensities of the ${ }^{15} \mathrm{~N}$ HSQC cross-peaks of the C-peptide would be explained by enhanced transverse relaxation rates in the bound state. Because of the high molecular mass of the OBdomain tetramer $(50 \mathrm{kDa})$, the C-peptide must be mostly in the unbound state to allow observation of ${ }^{15} \mathrm{~N}$ HSQC crosspeaks at the low sample concentrations used. In the limit of slow exchange, the small ${ }^{15} \mathrm{~N}$ HSQC cross-peaks observed for the C-peptide would arise from a small fraction of the Cpeptide in the unbound state, while the fraction in the bound state would be unobservable. To resolve the question of slow and fast exchange, we prepared a sample in which the methyl groups of methionines were labeled with ${ }^{13} \mathrm{C}$.

Methionine residues with $\left[{ }^{13} \mathrm{C}\right]$ methyl groups have been shown to yield readily observable ${ }^{13} \mathrm{C}$ HMQC cross-peaks in nondeuterated UvrB (a $75 \mathrm{kDa}$ protein), except for buried methionine residues that relax rapidly because of dipole-dipole interactions with spatially close protons. ${ }^{31}$ The SSB tetramer $(75 \mathrm{kDa})$ is thus suitable for this approach. The ${ }^{13} \mathrm{C}$ HMQC spectrum of SSB with $\left[{ }^{13} \mathrm{C}\right]$ methyl-labeled methionine indeed displayed four cross-peaks that could be attributed to SSB (Figure S5 of the Supporting Information). SSB contains five methionine residues, four in the OB-domain and one (Met169) preceding the C-peptide. The resonance of Met169 was assigned as follows. (i) Its cross-peak had the highest intensity, as expected for the residue with the highest mobility. (ii) Increasing $\mathrm{NaCl}$ concentrations shifted the cross-peak more strongly than the others (Figure 5A), as expected for a residue near the C-peptide (Figure S3A of the Supporting Information). (iii) The cross-peak moved toward the position of the unbound C-peptide in the complex with $(\mathrm{dT})_{35}$ (Figure 5C). (iv) Virtually the same salt-induced change in the ${ }^{1} \mathrm{H}$ NMR chemical shift was observed for the methyl group inserted sitespecifically at position 169 by reaction of the unlabeled SSB(M169C) mutant protein with $\left[{ }^{13} \mathrm{C}\right]$ methyl-methanethiosulfonate (MMTS) (Text S2 and Figure S6 of the Supporting Information).

The gradual shift of the resonance of Met169 with increasing salt concentrations toward the chemical shift of the unbound Cpeptide demonstrates that the exchange between the bound and free C-peptide is fast on the chemical shift time scale (microseconds to milliseconds). In addition, the peak heights of all cross-peaks of SSB increased, indicating increased mobility of the C-peptide in concert with its release from the SSB tetramer.

Titration of $\left[{ }^{13} \mathrm{C}\right]$ Methionine-Labeled SSB with $(\mathrm{dT})_{35}$ The chemical shift of Met169 in the unbound C-peptide can be gleaned from its chemical shift in the complex with ssDNA. In 
complete analogy to the titration experiment with ${ }^{15} \mathrm{~N}$-labeled protein (Figure 3 ), titration with $(\mathrm{dT})_{35}$ led to the appearance of new sets of signals (Figure 5C,D), where the signal of Met169 in the 1:1 complex appeared at almost the same chemical shift as the signal of the free C-peptide (observed in the $2: 1$ complex) and became taller than the signal in the absence of ssDNA. The similarity in chemical shifts of Met169 in the 2:1 and 1:1 complexes indicates that the C-peptides of all SSB subunits are already mostly in the unbound state in the $1: 1$ complex. The NMR line shapes of the 1:1 complex appear to be inhomogeneous, reflecting the different chemical environments of different C-peptides, but the line shape of Met169 in the 2:1 complex appears to be mostly homogeneous as expected for the more uniform environment of the unbound state of the C-peptide. In contrast, the signals of the other methionine residues are subject to significant line broadening, which is not unexpected as $(\mathrm{dT})_{35}$ breaks the symmetry in the tetramer and ssDNA may exchange between different conformations. The inhomogeneity of the NMR line shapes in the $1: 1$ complex was also confirmed by relaxation measurements of the SSB(M169C) mutant tagged with $\left[{ }^{13} \mathrm{C}\right]$ MMTS (Figures $\mathrm{S} 7$ and $\mathrm{S} 8$ of the Supporting Information).

Population of the C-Peptide in the Bound State at Low Salt Concentrations without ssDNA. Fast exchange between the bound and unbound C-peptide makes it possible to estimate the population of the bound state at low salt concentrations in the absence of ssDNA from NMR line widths. In the ${ }^{15} \mathrm{~N}$ HSQC spectrum without ssDNA, the total width of the ${ }^{1} \mathrm{H}$ NMR multiplets of any of the C-peptide amide resonances did not exceed $35 \mathrm{~Hz}$, which became $15 \mathrm{~Hz}$ in the 2:1 complex with $(\mathrm{dT})_{35}$. In the fast exchange limit, the relaxation rates are the population-averaged values of the bound and free states. ${ }^{32}$ As $15 \mathrm{~Hz}$ represents the line width in the unbound state, only $20 \mathrm{~Hz}$ of the line width observed in the absence of ssDNA can be attributed to exchange with the bound state. To estimate the line width in the bound state, we note that the tetramer of OB-domains has a molecular mass of $50 \mathrm{kDa}$ and the SSB tetramer has a mass of $75 \mathrm{kDa}$. Using HYDRONMR, ${ }^{33}$ the rotational correlation time $\left(\tau_{c}\right)$ of the OBdomain tetramer is calculated to be $\sim 32$ ns. For this $\tau_{\mathrm{c}}$ value, the transverse ${ }^{1} \mathrm{H}$ relaxation rates of amide protons are predicted to be $\sim 170 \mathrm{~s}^{-1}$. ${ }^{34}$ Allowing for a small increase in the $\tau_{\mathrm{c}}$ value caused by the presence of the flexible C-domains, we found the ${ }^{1} \mathrm{H}$ NMR line width could be estimated to be at least $60 \mathrm{~Hz}$. Therefore, at most one-third of the C-peptides can be bound to OB-domains, as otherwise the exchange broadening would exceed $20 \mathrm{~Hz}$. The population of the bound state could easily be lower, if the exchange is not in the limit of fast exchange or if the actual $\tau_{\mathrm{c}}$ value is larger because of intermolecular association. Notably, the population estimate would also be lower if the exchange between the bound and free C-peptide were slow on the chemical shift time scale, as the ${ }^{15} \mathrm{~N}$ HSQC cross-peaks of the C-peptide grow $\sim 6$-fold upon addition of $(\mathrm{dT})_{35}$ (Figure $\left.3 \mathrm{~B}\right)$. In conclusion, most of the $\mathrm{C}$ peptide is in the free state even in the absence of ssDNA.

EPR Spectroscopy. Using the SSB(M169C) mutant with the nitroxide radical MTSL attached at residue 169, we recorded EPR spectra of free SSB and SSB in complex with $(\mathrm{dT})_{35}$. The spectra of the $1: 1$ and 2:1 complexes were indistinguishable and in agreement with a highly mobile nitroxide radical (Figure 6). In contrast, each multiplet line in the spectrum of free SSB appeared as the sum of an intense
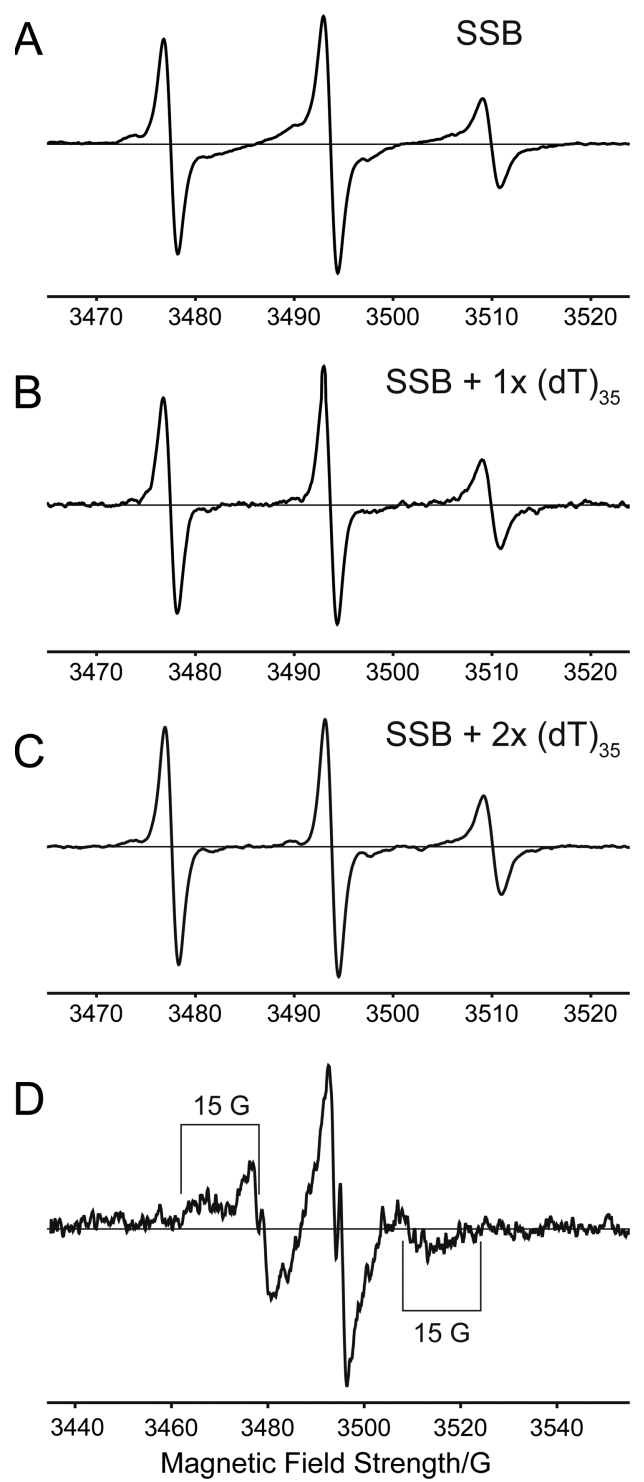

Figure 6. X-Band EPR spectra of SSB(M169C) with a nitroxide (MTSL) tag on residue 169 . The spectra of $10 \mu \mathrm{M}$ aqueous solutions of tetramer (A) without ssDNA, (B) with $(\mathrm{dT})_{35}$ present at a $1: 1$ molar ratio, and $(\mathrm{C})$ with $(\mathrm{dT})_{35}$ present at a 2:1 molar ratio were recorded at room temperature. (D) Difference spectrum calculated as the spectrum of free SSB in panel A minus the spectrum of the 2:1 complex of $(\mathrm{dT})_{35}$ and SSB in panel C, highlighting the broad component in the EPR spectrum. The hyperfine features of $\sim 15 \mathrm{G}$ (corresponding to a frequency interval of $\sim 40 \mathrm{MHz}$ ) indicate that the nitroxide is in the slow motion regime. Note the different scale of the axis in panel D.

narrow and a weak broad component. We attribute the narrow component to the unbound C-peptide and the broad component to the C-peptide bound to the OB-domain tetramer. Isolating the broad component from the spectrum of free SSB by subtracting the spectrum of the 2:1 complex revealed hyperfine features in line with expectations for the $\mathrm{C}$ peptide immobilized by binding to the OB-domain tetramer (Figure 6D). Observation of these hyperfine features is possible because of a rotational correlation time of the SSB tetramer of at least $32 \mathrm{~ns}$, corresponding to a rotation rate of $<30 \mathrm{MHz}$. The EPR spectra thus confirm that the C-peptide is overwhelmingly in the unbound state in free SSB, and there is no evidence that any $\mathrm{C}$-peptide-OB-domain interaction 
persists in the $1: 1$ complex. As the chemical nature of the tag could, in principle, interfere with the weak C-peptide-OBdomain interaction, we did not attempt to quantify the broad EPR component.

Release of the C-Peptide in the 1:1 Complex with ssDNA. As the exchange between the bound and unbound Cpeptide is fast, the chemical shift difference between the bound and free C-peptide can be taken as a measure of the bound and free populations. The chemical shift of the Met169 signal in the $1: 1$ complex is $0.074 \mathrm{ppm}$ downfield from its chemical shift in the absence of ssDNA and within $0.015 \mathrm{ppm}$ of the chemical shift of the unbound C-peptide, which is observed in the 2:1 complex (Figure 5C). Therefore, at most $20 \%$ of the C-peptide can be bound in the 1:1 complex, but as approximately twothirds of the C-peptides are already unbound in the absence of ssDNA, the actual limit on the population of the bound state in the $1: 1$ complex is two-thirds lower $(\sim 7 \%)$. By the same argument, $300 \mathrm{mM} \mathrm{NaCl}$ decreases the population of the $\mathrm{C}$ peptide in the bound state by more than half (Figure 5A).

Intertetramer Interactions. Intertetramer interactions are expected as the long flexible $\mathrm{C}$-domain can present the $\mathrm{C}$ peptide to OB-domains of different SSB tetramers in the same way as to the OB-domains of its own SSB tetramer. Indeed, the peak heights of all methionine methyl peaks more than doubled when the salt concentration increased from 10 to $300 \mathrm{mM}$ (Figure 5A), indicating that the rotational correlation time of the SSB tetramer decreases when the C-peptide no longer binds to the OB-domain with significant affinity. As salt increases the viscosity of aqueous solutions, the opposite effect would be expected if intertetramer interactions were absent.

Intermolecular Binding Affinity of the C-Peptide. To measure the binding affinity of the C-peptide for the OBdomain in an intermolecular experiment, we performed microscale thermophoresis (MST) experiments using a fluorescently tagged SSB mutant in which the eight C-terminal residues were deleted to allow binding of a synthetic peptide (termed "SSB-Ct", amino acid sequence of PSNEPPMDFDDDIPF) that comprised the C-peptide. The fluorescein 5maleimide tag was attached to cysteine incorporated at position 43 of SSB $\Delta$ C 8 . To minimize the possible interference of the fluorescein tag with the binding of SSB-Ct, tagged SSB $\Delta$ C8(K43C) was allowed to equilibrate by subunit exchange with a 25 -fold excess of "wild-type" SSB $\Delta \mathrm{C} 8^{9}$ to produce a population of tetramers in which a maximum of one subunit would be fluorescent. Using a simple two-state model to fit the MST measurements, the data indicated a dissociation constant of 13 $\pm 3 \mathrm{mM}$ (Figure 7). Repeating these measurements with $\mathrm{SSB} \Delta \mathrm{C} 62(\mathrm{~K} 43 \mathrm{C} /$ tagged $) / \mathrm{SSB} \Delta \mathrm{C} 62$ yielded the same binding affinity $(11 \pm 2 \mathrm{mM})$, showing that the rest of the C-domain (residues 113-169) interferes little with the binding of SSB-Ct to the OB-domain.

The addition of more and more SSB-Ct molecules to the fulllength SSB tetramer would be expected to change the response in the MST experiment in an unpredictable manner, so the assumption of a two-state model is likely to be reliable only for low ligand concentrations. Nevertheless, when we conducted the MST experiment with a 1:25 mixture of fluorescein-tagged SSB(K43C) and SSB, the fit to a simple two-state model yielded an apparent binding affinity of $19 \pm 5 \mathrm{mM}$, excluding the data point at the highest SSB-Ct concentration (103 mM) from the fit (Figure 7). The deviation of the data from the fit curve at $1-10 \mathrm{mM}$ SSB-Ct suggests that the C-peptide in fulllength SSB competes with SSB-Ct to some degree for binding

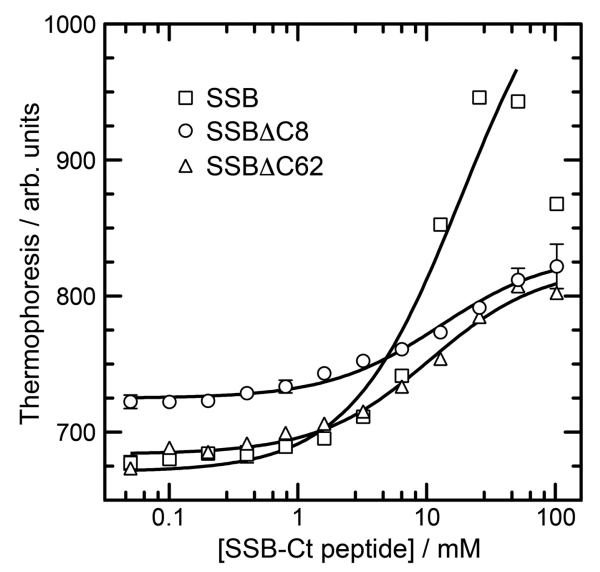

Figure 7. MST measurements of the binding affinity of SSB-Ct for the $\mathrm{OB}$-domain tetramer. The measurements were performed at room temperature with a fluorophore conjugated with the cysteine residue in K43C mutants of SSB. The tetramer concentration was $1.6 \mu \mathrm{M}$ in a buffer of $10 \mathrm{mM}$ Tris- $\mathrm{HCl}(\mathrm{pH} \mathrm{7.6)}$ ) and $0.01 \%$ Tween 20. Binding isotherms show MST responses vs the concentration of SSB-Ct. Each measurement was made in duplicate (error bars show the standard error of the mean). Measurements used full-length SSB ( $\square$ ), SSB $\Delta$ C8 $(\mathrm{O})$, or SSB $\Delta \mathrm{C} 62$ [also known as $\operatorname{SSB}^{*}{ }^{*}(\triangle)$ ]. Dissociation constants $\left(K_{\mathrm{D}}\right)$ were determined by regression, assuming a simple twostate Langmuir binding system. The fit curves $(-)$ correspond to $K_{\mathrm{D}}$ values of $18.8 \mathrm{mM}$ (SSB), $12.7 \mathrm{mM}$ (SSB $\Delta \mathrm{C} 8$ ), and $10.6 \mathrm{mM}$ (SSB $\Delta$ C62). The fit for SSB excluded the data point at $103 \mathrm{mM}$. The deviation of the experimental data from the fit curve for SSB at 1-10 $\mathrm{mM}$ SSB-Ct is indicative of competition with the C-peptide in fulllength SSB. The similarity of the dissociation constants suggests that the $\mathrm{C}$-domain residues preceding the $\mathrm{C}$-peptide make no significant contribution to the binding of SSB-Ct.

to the OB-domain, but the competition does not appear to be strong.

Intramolecular Concentration Enhancement. While the intrinsic affinity of the C-peptide for the OB-domain is weak, binding is promoted by the intramolecular nature of the interaction, which increases the local concentration of the Cpeptide near the OB-domain. To obtain a quantitative estimate of this effect, we simulated the probability that the C-peptide comes within $5 \AA$ of its binding site on the OB-domain, assuming that the intervening polypeptide chain of the $\mathrm{C}$ domain is fully flexible.

The simulation represented the polypeptide chain of the Cdomain by a Gaussian chain of statistical chain segments that are randomly oriented with respect to each other (see Text S3 of the Supporting Information for details). Interestingly, for a given starting point of the random walk in one of the $\mathrm{OB}$ domains in the tetramer, the probabilities of approaching the $\mathrm{C}$ peptide binding site of any of the four different OB-domains in the tetramer varied by no more than 2 -fold.

To estimate the increase in the effective local concentration of the C-peptide, the probability of binding found by the Gaussian chain model must be compared to the probability of finding the C-peptide in the binding site if the peptide concentration is uniform as in the affinity measurements performed by MST with SSB-Ct. The space sampled by the Cdomain in full-length SSB is defined by a sphere with a radius given by the fully extended chain $(7 \times 35 \AA=245 \AA)$. The probability of finding SSB-Ct in a sphere with a radius of $5 \AA$ is given by the volume ratio of the spheres with radii of 5 and 245 $\AA$. This probability is $10-20$-fold lower (depending on the 
binding site in the tetramer) than the probability of binding calculated with the Gaussian chain model for the SSB tetramer. Therefore, the covalent linker between the C-peptide and the OB-domain in full-length SSB increases the affinity of binding approximately 10-20-fold compared with the intermolecular MST measurements.

One molecule located in the space of a sphere with a radius of $245 \AA$ corresponds to a concentration of $\sim 30 \mu \mathrm{M}$. This is small compared with the $K_{\mathrm{D}}$ measured by MST [10-20 mM (Figure 7)]. Even if the C-terminus in full-length SSB has a 20fold higher statistical chance of being close to the binding site of its own OB-domain and considering it could bind to any of four OB-domains, these calculations suggest that further enrichment of the local concentration of the C-peptide by electrostatic attractions is required for a significant population of the bound state, in agreement with the salt effects reported here.

\section{DISCUSSION}

C-Domain-OB-Domain Interactions. Our study demonstrates that, in solution and at neutral $\mathrm{pH}$, the $\mathrm{C}$-domain is highly mobile in the SSB tetramer and that its C-terminal segment (the $\mathrm{C}$-peptide) is in fast exchange between a free state and a bound state in which it interacts with the OB-domain tetramer in competition with ssDNA. The flexible C-domain is sufficiently long for the C-peptide to bind to any of the OBdomains of the tetramer with similar probability, and there is also evidence of intermolecular interactions with other SSB tetramers. As demonstrated by our NMR and EPR results, the population of the bound state is low, leaving most of the Cpeptides available for binding to SSB-binding proteins even in the absence of ssDNA. In view of the weakness of the Cpeptide-OB-domain interaction, it is clear that binding of ssDNA to SSB is not a prerequisite for making the C-peptide available for binding to SSB-binding proteins. Nonetheless, we find that binding of $(\mathrm{dT})_{35}$ to two of the OB-domains leads to nearly complete release of C-peptides from the other two OBdomains of the tetramer, in agreement with previous conclusions derived from comparisons of ssDNA binding affinities for full-length SSB and SSB $\Delta C 8{ }^{23}$ The increased level of dissociation of C-peptides from the OB-domains upon binding of ssDNA may be driven by electrostatic repulsion between ssDNA and the C-peptide. Our data indicate that in the 1:1 complex of an SSB tetramer and one molecule of $(\mathrm{dT})_{35},<10 \%$ of the C-peptides remain bound to OB-domains.

Previous evidence of the interaction of the C-peptide with the OB-domain has been indirect and ambiguous with respect to the strength of this interaction. For example, at intermediate salt concentrations (100 and $200 \mathrm{mM} \mathrm{NaCl}),(\mathrm{dA})_{35}$ was found to bind more tightly to SSB $\Delta$ C 8 than to full-length SSB by $1-2$ orders of magnitude, but at a low salt concentration $(20 \mathrm{mM}$ $\mathrm{NaCl}$ ), binding to SSB appeared to be stronger than that to $\mathrm{SSB} \Delta \mathrm{C} 8{ }^{23}$ In another example, an ultracentrifugation study of the complex between SSB and subunit $\chi$ of DNA polymerase III at $5 \mathrm{mM} \mathrm{NaCl}$ indicated that the stability of the complex was enhanced almost 20 -fold in the presence of poly $(\mathrm{dT}) .^{35}$ As $\chi$ binds to the C-terminus of SSB, this result suggests an increased availability of the C-peptide in the presence of ssDNA, but it is unclear how much the effect was influenced by the presence of $670 \mathrm{mM}$ glycerol. In other experiments using SSB and SSB $\Delta$ C8, the presence of the C-peptide was found to exert only subtle effects on the interconversion between $(\mathrm{SSB})_{35}$ and $(\mathrm{SSB})_{65}$ binding modes. ${ }^{36}$ An early study pointed out that binding of SSB to poly $(\mathrm{dT})$ increases the sensitivity of the C-domain toward proteolysis with chymotrypsin. ${ }^{8}$ Rather than indicating release of the C-peptide by ssDNA, this result could also be explained by the enhanced attraction of chymotrypsin, which is overall positively charged, to the overall negatively charged SSB-ssDNA complex.

Role of Electrostatic Charge. E. coli SSB contains 18 aspartate and glutamate residues and 16 arginine and lysine residues in each subunit. Therefore, and significantly because of the C-peptide, the overall charge of SSB is negative at neutral $\mathrm{pH}$. In light of the limited strength of the interaction of the Cpeptide with the OB-domain, it is interesting to consider the role of electrostatic effects in the interactions made by SSB. If electrostatic effects were decisive, this could explain some of the relative binding affinities observed for the PriA helicase (an overwhelmingly positively charged protein that binds to the $\mathrm{C}$ peptide) and DNA polymerase III subunit $\chi$ (a protein with a single overall negative charge that also binds to the C-peptide). Thus, at $200 \mathrm{mM} \mathrm{NaCl}$, where quantitative data are available for binding to SSB and the SSB- $(\mathrm{dT})_{70}$ complex, PriA was found to bind $\sim 10$-fold more strongly to the $\mathrm{SSB}-(\mathrm{dT})_{70}$ complex than to SSB, whereas $\chi$ binds to the SSB-(dT) ${ }_{70}$ complex and SSB with similar affinities. ${ }^{37}$

We note that $E$. coli uses glutamate rather than chloride to regulate osmotic pressure; i.e., the concentration of chloride in the cytosol is low, while the concentration of glutamate can increase to $200 \mathrm{mM}$ on growth in highly osmotic media. ${ }^{30}$ Our study shows that, in contrast to $\mathrm{NaCl}$, potassium glutamate does not promote the release of the C-peptide. Therefore, physiological conditions for SSB are best approximated by low concentrations of $\mathrm{NaCl}$.

SSB Polymerization. Our data show that C-peptide-OBdomain interactions are weakened following the binding of ssDNA. Specifically, following binding of 1 equiv of $(\mathrm{dT})_{35}$ to form the $1: 1$ complex in the (SSB $)_{35}$ binding mode, $<10 \%$ of the C-peptides remain bound. This weak residual C-peptideOB-domain interaction could nonetheless assist the formation of the dense SSB polymer on ssDNA observed by EM at high SSB concentrations and low ionic strengths. ${ }^{38,39}$

The Gaussian chain model indicated that the C-domain is sufficiently long that the C-peptide can occupy binding sites in any of the four OB-domains in the tetramer with similar ease. The C-domain can thus be regarded as a long flexible tether, capable of projecting the C-peptide far out into solution for binding to SSB-binding proteins and other SSB tetramers, as well as allowing binding interactions with different OB-domains of its own tetramer, shown previously to stabilize the tetrameric state. ${ }^{9}$ In addition, we observed intertetramer interactions at low salt concentrations, suggesting that C-peptides can also bind to the OB-domains of other SSB tetramers. As the proximity of SSB tetramers on ssDNA strands increases the concentration of OB-domains within reach of any given Cpeptide, more binding opportunities are created for C-peptides, which can loosely tie together neighboring SSB tetramers. As observed previously, however, C-peptide-OB-domain interactions are not the only link between two neighboring SSB tetramers, as SSB tetramers cluster together even under conditions where an excess of ssDNA favors the (SSB) 65 mode, in which all OB-domains are occupied by ssDNA. ${ }^{40}$ For example, conserved crystal contacts between adjacent SSB tetramers suggest that filaments of SSB tetramers can arise from interactions between OB-domains. ${ }^{1}$ 
Release of C-Peptides as a Signal of ssDNA Binding. This study shows that most of the C-peptides are already dissociated from OB-domains in the absence of ssDNA or salt, so that binding of ssDNA leads only to a modest increase in the population of free C-peptides. Furthermore, this increase is attenuated by the additional binding opportunities offered by vacant OB-domains of neighboring SSB tetramers on the ssDNA in the (SSB $)_{35}$ mode. It is thus difficult to attribute a decisive role to the release of C-peptides from OB-domains upon binding of ssDNA as a signal for the binding of other ssDNA processing proteins. Long-range electrostatic effects rather than engagement of the C-peptide by the OB-domain may be a more important aspect of how the C-peptide attenuates the affinity of SSB for $\operatorname{ssDNA}^{23}$ and how ssDNA enhances the affinity of SSB for SSB-binding proteins. ${ }^{37}$

Measuring Intramolecular Binding Affinities. This study addresses the difficult problem of determining the populations of bound and free states in a fast intramolecular equilibrium, where the fully bound state is neither observable nor experimentally accessible. The problem was overcome by estimating the parameters of the fully bound state. In contrast to the fast exchange regime in NMR, the equilibrium was found to be in the slow exchange regime on the time scale of EPR spectroscopy, but the EPR measurements could potentially have been affected by the necessity of modifying the protein with an EPR spin-label. Finally, we performed conventional affinity measurements by severing the link between the interaction partners and using titration experiments with increasing concentrations of one of the partners. These experiments not only required mutation of the protein but also were affected by the high concentrations required to detect the weak binding affinities. While all three techniques demonstrate the weakness of the C-peptide-OB-domain interaction in E. coli SSB, the NMR results provide the most reliable picture of the populations of the bound and free states of the C-peptide.

\section{ASSOCIATED CONTENT}

\section{S Supporting Information}

Experimental procedures, plasmid construction, protein expression and purification, NMR spectra, chemical shift data, titration of ${ }^{13} \mathrm{C}$ MMTS SSB with $\mathrm{NaCl}$ and $(\mathrm{dT})_{35}$, relaxation data and pulse sequence, and Gaussian chain model calculations. This material is available free of charge via the Internet at http://pubs.acs.org.

\section{AUTHOR INFORMATION}

\section{Corresponding Author}

*Telephone: +61-2-61256507. E-mail: gottfried.otting@anu. edu.au.

\section{Author Contributions}

X.-C.S., Y.W., and H.Y. contributed equally to this work.

\section{Funding}

This work was supported by the Australian Research Council (DP0877658 and DP0984797 to N.E.D. and DP110102737 and DP120100561 to G.O.) and the National Science Foundation of China (21073101 to X.-C.S.).

\section{Notes}

The authors declare no competing financial interest.

\section{ACKNOWLEDGMENTS}

We thank Professor Ron Pace for helpful discussions regarding the EPR data, Professor Joel P. Mackay for access to the MST instrument, Penelope Lilley for technical assistance, and Dr. Christopher Love for construction of plasmid pCL547.

\section{ABBREVIATIONS}

SSB, ssDNA-binding protein; OB, oligonucleotide (and oligosaccharide) binding; ssDNA, single-stranded DNA; MMTS, methyl-methanethiosulfonate; MTSL, S-(2,2,5,5-tetramethyl-2,5-dihydro-1H-pyrrol-3-yl)methyl methanesulfonothioate; MST, microscale thermophoresis; NMR, nuclear magnetic resonance; EPR, electron paramagnetic resonance; ESI-MS, electrospray ionization mass spectrometry.

\section{REFERENCES}

(1) Shereda, R. D., Kozlov, A. G., Lohman, T. M., Cox, M. M., and Keck, J. L. (2008) SSB as an organizer/mobilizer of genome maintenance complexes. Crit. Rev. Biochem. Mol. Biol. 43, 289-318.

(2) Marceau, A. H. (2012) Functions of single-strand DNA-binding proteins in DNA replication, recombination and repair. Methods Mol. Biol. 922, 1-21.

(3) Meyer, R. R., and Laine, P. S. (1990) The single-stranded DNAbinding protein of Escherichia coli. Microbiol. Rev. 54, 342-380.

(4) Lohman, T. M., and Ferrari, M. E. (1994) Escherichia coli singlestranded DNA-binding protein: Multiple DNA-binding modes and cooperativities. Annu. Rev. Biochem. 63, 527-570.

(5) Suck, D. (1997) Common fold, common function, common origin? Nat. Struct. Biol. 4, 161-165.

(6) Bujalowski, W., Overman, L. B., and Lohman, T. M. (1988) Binding mode transitions of Escherichia coli single strand binding protein-single-stranded DNA complexes. Cation, anion, $\mathrm{pH}$, and binding density effects. J. Biol. Chem. 263, 4629-4640.

(7) Overman, L. B., and Lohman, T. M. (1994) Linkage of pH, anion and cation effects in protein-nucleic acid equilibria. Escherichia coli SSB protein-single stranded nucleic acid interactions. J. Mol. Biol. 236, $165-178$.

(8) Williams, K. R., Spicer, E. K., LoPresti, M. B., Guggenheimer, R. A., and Chase, J. W. (1983) Limited proteolysis studies on the Escherichia coli single-stranded DNA binding protein. Evidence for a functionally homologous domain in both the Escherichia coli and T4 DNA binding proteins. J. Biol. Chem. 258, 3346-3355.

(9) Mason, C. E., Jergic, S., Lo, A. T. Y., Wang, Y., Dixon, N. E., and Beck, J. L. (2013) Escherichia coli single-stranded DNA-binding protein: NanoESI-MS studies of salt-modulated subunit exchange and DNA binding transactions. J. Am. Soc. Mass Spectrom. 24, 274-285.

(10) Lohman, T. M., and Bujalowski, W. (1988) Negative cooperativity within individual tetramers of Escherichia coli single strand binding protein is responsible for the transition between the $(\mathrm{SSB})_{35}$ and (SSB $)_{56}$ DNA binding modes. Biochemistry 27, 22602265.

(11) Yang, C., Curth, U., Urbanke, C., and Kang, C. H. (1997) Crystal structure of human mitochondrial single-stranded DNA binding protein at 2.4 Å resolution. Nat. Struct. Biol. 4, 153-157.

(12) Raghunathan, S., Ricard, C. S., Lohman, T. M., and Waksman, G. (1997) Crystal structure of the homo-tetrameric DNA binding domain of Escherichia coli single-stranded DNA-binding protein determined by multiwavelength X-ray diffraction on the selenomethionyl protein at $2.9 \AA$ resolution. Proc. Natl. Acad. Sci. U.S.A. 94, $6652-6657$.

(13) Raghunathan, S., Kozlov, A. G., Lohman, T. M., and Waksman, G. (2000) Structure of the DNA binding domain of E. coli SSB bound to ssDNA. Nat. Struct. Biol. 7, 648-652.

(14) Matsumoto, T., Morimoto, Y., Shibata, N., Kinebuchi, T., Shimamoto, N., Tsukihara, T., and Yasuoka, N. (2000) Roles of functional loops and C-terminal segments of a single-stranded DNA 
binding protein elucidated by X-ray structural analysis. J. Biochem. 127, 329-335.

(15) Savvides, S. N., Raghunathan, S., Fütterer, K., Kozlov, A. G., Lohman, T. M., and Waksman, G. (2004) The C-terminal domain of full-length E. coli SSB is disordered even when bound to DNA. Protein Sci. 13, 1942-1947.

(16) Chan, K.-W., Lee, Y.-J., Wang, C.-H., Huang, H., and Sun, Y.-J. (2009) Single-stranded DNA-binding protein complex from Helicobacter pylori suggests an ssDNA-binding surface. J. Mol. Biol. 388, 508-519.

(17) Kaushal, P. S., Singh, P., Sharma, A., Muniyappa, K., and Vijayan, M. (2010) X-ray and molecular-dynamics studies on Mycobacterium leprae single-stranded DNA-binding protein and comparison with other eubacterial SSB structures. Acta Crystallogr. D66, 1048-1058.

(18) Antony, E., Weiland, E. A., Korolev, S., and Lohman, T. M. (2012) Plasmodium falciparum SSB tetramer wraps single-stranded DNA with similar topology but opposite polarity to E. coli SSB. J. Mol. Biol. 420, 269-283.

(19) Yadav, T., Carrasco, B., Myers, A. R., George, N. P., Keck, J. L., and Alonso, J. C. (2012) Genetic recombination in Bacillus subtilis: A division of labor between two single-strand DNA-binding proteins. Nucleic Acids Res. 40, 5546-5559.

(20) Cheng, Z., Caillet, A., Ren, B., and Ding, H. (2012) Stimulation of Escherichia coli DNA damage inducible DNA helicase DinG by the single-stranded DNA binding protein SSB. FEBS Lett. 586, 38253830 .

(21) Lu, D., and Keck, J. L. (2008) Structural basis of Escherichia coli single-stranded DNA-binding protein stimulation of exonuclease I. Proc. Natl. Acad. Sci. U.S.A. 105, 9169-9174.

(22) Curth, U., Genschel, J., Urbanke, C., and Greipel, J. (1996) In vitro and in vivo function of the C-terminus of Escherichia coli singlestranded DNA binding protein. Nucleic Acids Res. 24, 2706-2711.

(23) Kozlov, A. G., Cox, M. M., and Lohman, T. M. (2010) Regulation of single-stranded DNA binding by the $\mathrm{C}$ termini of Escherichia coli single-stranded DNA-binding (SSB) protein. J. Biol. Chem. 285, 17246-17252.

(24) Marintcheva, B., Marintchev, A., Wagner, G., and Richardson, C. C. (2008) Acidic C-terminal tail of the ssDNA-binding protein of bacteriophage $\mathrm{T} 7$ and ssDNA compete for the same binding surface. Proc. Natl. Acad. Sci. U.S.A. 105, 1855-1860.

(25) Antony, E., Weiland, E., Yuan, Q., Manhart, C. M., Nguyen, B., Kozlov, A. G., McHenry, C. S., and Lohman, T. M. (2013) Multiple Cterminal tails within a single E. coli SSB homotetramer coordinate DNA replication and repair. J. Mol. Biol. 425, 4802-4819.

(26) Shishmarev, D., Wang, Y., Mason, C. E., Su, X.-C., Oakley, A. J., Graham, B., Huber, T., Dixon, N. E., and Otting, G. (2014) Intramolecular binding mode of the C-terminus of E. coli singlestranded DNA binding protein (SSB) determined by nuclear magnetic resonance spectroscopy. Nucleic Acids Res. 42, 2750-2757.

(27) Potapov, A., Yagi, H., Huber, T., Jergic, S., Dixon, N. E., Otting, G., and Goldfarb, D. (2010) Nanometer-scale distance measurements in proteins using $\mathrm{Gd}^{3+}$-spin labeling. J. Am. Chem. Soc. 132, 90409048.

(28) Farrow, N. A., Muhandiram, R., Singer, A. U., Pascal, S. M., Kay, C. M., Gish, G., Shoelson, S. E., Pawson, T., Forman-Kay, J. D., and Kay, L. (1994) Backbone dynamics of a free and phosphopeptidecomplexed Src homology 2 domain studied by ${ }^{15} \mathrm{~N}$ NMR relaxation. Biochemistry 33, 5984-6003.

(29) Goddard, T. D., and Kneller, D. G. (2010) Sparky 3, University of California, San Francisco.

(30) Record, M. T., Jr., Anderson, C. F., Mills, P., Mossing, M., and Roe, J. (1985) Ions as regulators of protein-nucleic acid interactions in vitro and in vivo. Adv. Biophys. 20, 109-135.

(31) DellaVecchia, M. J., Merritt, W. K., Peng, Y., Kirby, T. W., DeRose, E. F., Mueller, G. A., Van Houten, B., and London, R. E. (2007) NMR analysis of $\left[\right.$ methyl $\left.{ }^{13} \mathrm{C}\right]$ methionine UvrB from Bacillus caldotenax reveals UvrB-domain 4 heterodimer formation in solution. J. Mol. Biol. 373, 282-295.
(32) Su, X. C., Jergic, S., Ozawa, K., Burns, N. D., Dixon, N. E., and Otting, G. (2007) Measurement of dissociation constants of highmolecular weight protein-protein complexes by transferred ${ }^{15} \mathrm{~N}$ relaxation. J. Biomol. NMR 38, 65-72.

(33) García de la Torre, J., Huertas, M. L., and Carrasco, B. (2000) HYDRONMR: Prediction of NMR relaxation of globular proteins from atomic-level structures and hydrodynamic calculations. J. Magn. Reson. 147, 138-146.

(34) Kontaxis, G., Clore, G. M., and Bax, A. (2000) Cross-correlation effects and measurements of one-bond couplings in proteins with short transverse relaxation times. J. Magn. Reson. 143, 184-196.

(35) Witte, G., Urbanke, C., and Curth, U. (2003) DNA polymerase III $\chi$ subunit ties single-stranded DNA binding protein to the bacterial replication machinery. Nucleic Acids Res. 31, 4434-4440.

(36) Roy, R., Kozlov, A. G., Lohman, T. M., and Ha, T. (2007) Dynamic structural rearrangements between DNA binding modes of E. coli SSB protein. J. Mol. Biol. 369, 1244-1257.

(37) Kozlov, A. G., Jezewska, M. J., Bujalowski, W., and Lohman, T. M. (2010) Binding specificity of Escherichia coli single-stranded DNA binding protein for the $\chi$ subunit of DNA pol III holoenzyme and PriA helicase. Biochemistry 49, 3555-3566.

(38) Griffith, J. D., Harris, L. D., and Register, J., III (1984) Visualization of SSB-ssDNA complexes active in the assembly of stable RecA-DNA filaments. Cold Spring Harbor Symp. Quant. Biol. 49, 553559.

(39) George, N. P., Ngo, K. V., Chitteni-Pattu, S., Norais, C. A., Battista, J. R., Cox, M. M., and Keck, J. L. (2012) Structure and cellular dynamics of Deinococcus radiodurans single-stranded DNA (ssDNA)binding protein (SSB)-DNA complexes. J. Biol. Chem. 287, 2212322132.

(40) Chrysogelos, S., and Griffith, J. (1982) Escherichia coli singlestrand binding protein organizes single-stranded DNA in nucleosomelike units. Proc. Natl. Acad. Sci. U.S.A. 79, 5803-5807. 